\title{
SOCS Wayfinder: Using a Low Cost Solution for Geolocation and Pathfinding Indoors
}

\author{
Maxim K. Gorshkov \\ School of Computer Science \\ Faculty of Science \\ McGill University
}

\author{
Laurie Hendren \\ School of Computer Science \\ Faculty of Science \\ McGill University
}

\begin{abstract}
Navigation indoors can be difficult, especially for visitors or newcomers in public institutions such as university buildings, hospitals, and airports. Although many solutions exist for outdoor geolocation and wayfinding, through Global Position Systems (GPS), a similar standard doesn't exist for indoor navigation. The SOCS Wayfinder platform establishes a novel low cost solution for indoor navigation using Near Field Communication (NFC) Stickers and an Android application.
\end{abstract}

SOCS Wayfinder facilitates navigation inside the McConnell building to posters located at key locations. Once a user enters the building, they can download the application at any poster. Afterwards, the user chooses from popular points of interest such as bathrooms, lecture halls, and administrative offices and the application calculates the optimal route. A detailed map shows the blueprints of the building with all points of interests and adjacent buildings marked. Differently abled users can select a route and destinations with handicap access, as well as high contrast colours and patterns for the map. Should a user stray from the optimal route, a new route is recalculated immediately.

Quick user surveys ${ }^{1}$ and tracking of any individual scan assists in gathering valuable data for not only improvement of software, but also as a tool to improve building infrastructure. All of the features in this paper have been carefully implemented and tested with students, staff, and visitors.

\section{General Terms}

Indoor Navigation, Near Field Communication

\section{Keywords}

NFC Stickers, Geolocation, Wayfinding, Pathfinding

\section{INTRODUCTION}

Global Positioning Systems (GPS) are becoming ever more common for navigation in outdoor environments. There are

\footnotetext{
${ }^{1}$ This research has been approved by the McGill Research Ethics Board. REB File 121-0816.
}

significantly fewer solutions for navigation indoors, especially in public institutions, such as university buildings and hospitals. The purpose of this project was to investigate a low cost solution for indoor geolocation and pathfinding.

Initially, the project was created in the context of the MUHC Glen hospital, and the Cedars Cancer Centre. The centre is currently creating an application allowing patients to check in for their radiation appointments and to show the location of their treatments. A natural extension is to be able to wayfind from the waiting rooms to the treatment room, and around the hospital in general. Since the Glen hospital is a mega-hospital which amalgamated 3 previous hospitals and centres, it is impractical to know where a patient or a user is, without some sort of tool.

We decided to approach the problem on a smaller scale and use 3 floors of the McConnell Engineering building, belonging to the School of Computer Science. This way we were able to investigate the challenges of developing such a solutions before its implementation on a larger scale. McConnell itself, is also confusing to visitors, especially if it is the first time someone is in the building. For example, not every floor has bathrooms for both Women and Men, most major lecture theatres span between two floors thus allowing someone to enter from either of these floors, as well as the inconsistent numbering of rooms in some places of the building.

The SOCS Wayfinder Android application with the back-end stack helps a user find points of interest using NFC (Near Field Communication) stickers for wayfinding. Once a user finds the closest stickers, they enter the destination and the application calculates the shortest route. A user can scan stickers along the way to get step by step instructions on where to go. Each instruction is humanized and refers to points on reference unique to each route. If a user strays from the suggested route, the application recalculates the shortest route.

In our implementation we focus on wayfinding to specific points of interest: lecture theatres, bathrooms, administrative offices, and adjacent building entrances. We strategically placed stickers at key locations on each of the three floors to allow simple navigation when traversing the floor, as well as changing floors. The points of interest and paths take into account whether users require special accessible options, and the implementation allows 
for showing maps in different forms for users who have varying colour deficiencies.

Through our investigation, we wanted to find a way to feasibly implement such a solution without expensive equipment. Even though many different solutions such as iBeacons, WiFi signal triangulation, and QR codes exist, we focused on Near Field Communication (NFC) stickers. In addition to being a low cost solution, it also allowed us to evaluate a relatively new and novel technology in this domain. As each sticker is unique and holds just enough information to communicate with a mobile application, many functions are performed in the sophisticated back-end of our implementation.

The main contributions of this project are: investigation of NFC stickers as viable geolocation tools, development of a robust platform for geolocation and wayfinder which is easily extensible, identifying confusing areas in the McConnell building which require an extra tool, designing map representations to allow for users with varying levels of colour blindness, implementing instantaneous feedback to evaluate our calculated route.

The important features of the SOCS Wayfinder application include the extensibility to iOS and other platforms as a result of the technology used, ability to incorporate QR codes to support more devices, functionality to see and manipulate multiple floors of the building on-screen, humanized instructions that are specific to each set of points, and data collection that happens at each scan and at the end of each route.

This paper starts with the discussion of the technology behind NFC stickers. We then present our implementation of the application and back-end. Finally, we discuss related work, our contributions as well as how our implementation can be extended with future work.

\section{BACKGROUND}

Figure 1 shows an example of an NFC sticker used throughout this project [1 2]. On the left is how the user sees the sticker on an associated poster, on the right we show the coil which is under the decorative sticker. The general layout of an NFC sticker includes 2 main areas: an antenna and a chip. The antenna is inducted by a reader, in our case the phone. The chip actually holds the data which gets transmitted when it receives power. Figure 2 shows a simplified exchange between the application and the NFC sticker.
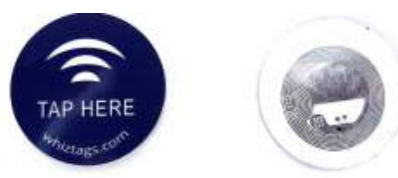

Fig. 1. NFC NTAG213 Chip Tag

Because of the simplicity of the NFC sticker, and the fact that it doesn't have a battery of its own, the lifetime of the sticker is many years. Furthermore, it is waterproof and inexpensive at between 10 and 70 cents a piece. This makes it a great choice for our project. However, it has a huge disadvantage since NFC stickers are only supported by some Android devices, and by no Apple devices. Most modern phones have NFC capabilities, but, iPhones do not allow for passive NFC communication, like these stickers use. Since
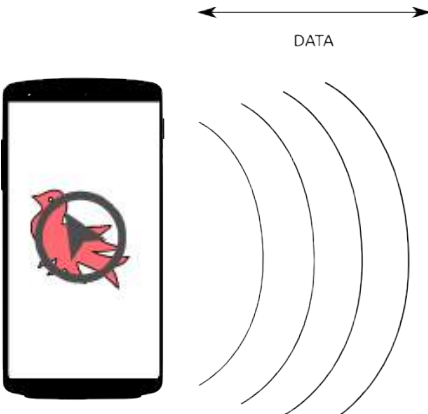

socs Wayfinder

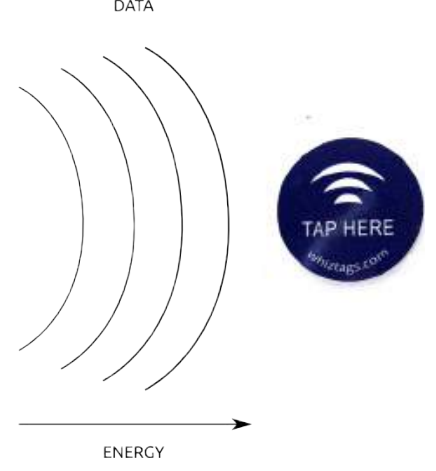

Fig. 2. Scanning NFC stickers with SOCS Wayfinder Application

iPhones make up a large portion of the mobile market, the most pertinent next step would be to incorporate a technology which is completely device and manufacturer neutral. In future work, we discuss QR codes as a way to incorporate devices which do not support NFC. The rest of the platform, the back-end, will not need to change, only the application will have to be modified to allow for the use of the camera and scanning functionality.

\section{APPLICATION OVERVIEW}

Figure 3 shows the conceptual flowchart of the SOCS Wayfinder application. Figure 4 shows how each of these phases would look to a user. When a user first downloads the application, the first run requires the user to view a tutorial, denoted (A) in the digram, on how to use the application. This gives a brief overview of the main features of the application as well as shows the proper way to scan NFC stickers. Once a user has looked through the application, they must complete the consent form in order to use the application (B). When they are ready to start scanning stickers, the final "Next" button takes the user to the scanning page, denoted (C) in the flowchart.

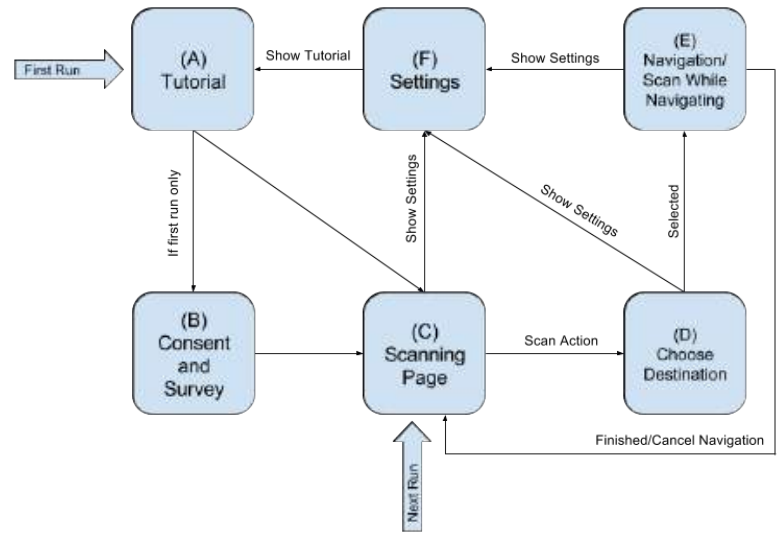

Fig. 3. Conceptual flowchart of the application

Once the user has scanned the nearest NFC sticker, the location of the user shows up overlaying the blueprints of the building. In our conceptual flowchart, the user is now at point (D). Note that the user may now expand the different types of point of interest 

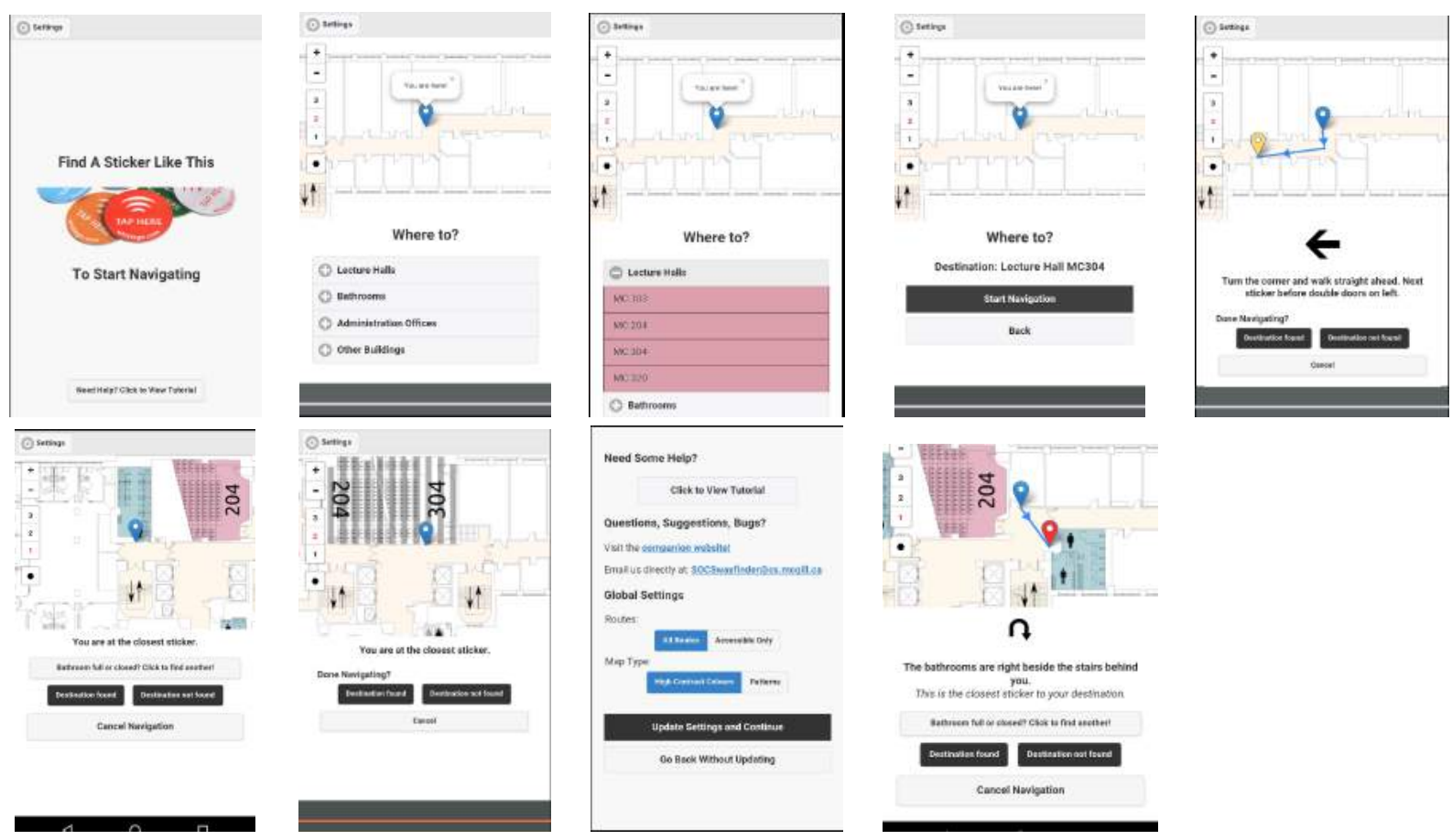

Fig. 4. Relative to the flowchart, the top row shows the following phases in order, from left to right: (C), (D), (D), (D), (E). On the bottom row, from left to right: $(\mathrm{E}),(\mathrm{E}),(\mathrm{F})$ with the rightmost image showing the special bathroom button.

categories to see all of the places to which they may navigate. On the navigational map at all times, the user may zoom in and out using the top left buttons, as well as through the pinching gesture. The numbered buttons represent the floors supported by the application. The highlighted coloured button is the current floor. Clicking different buttons changes the blueprints. If the user is currently in navigation mode, all waypoint stickers for the current floor will be shown. Finally, the button with the circular icon takes the user back to the current sticker and shows the relevant floor.

Once a user has chosen to expand a menu, the specific options are shown for the category. A user would then select a specific destination, which is then confirmed and navigation can begin, depicted as (E) in our flowchart. At this point, a user seems the direction in which they should go, relative to the poster that they scanned. For example, if the arrow is to the left, the user should physically turn their body to the left and navigate using the instructions. All instructions on the screen are human-centric and are intended to provide enough details to remove ambiguity in navigation. This approach allows the user to combine the instructions with the arrowed line draw over the blueprints to allow for efficient and clear directions.

Once a user has come to the closest sticker in the calculated route, the instructions and navigational aides finish. A final message is displayed. Once a user finishes navigating, they are taken back to the scanning page, and the user can then navigate to their next destination. At any point between opening the scanning page and finishing navigation, the user can select the settings button in the top bar. This takes them to the settings area with the opportunity to change global settings and to get the information of the researchers.
In the flow chart this is depicted as $(\mathrm{F})$.

Special circumstances can lead to other screens as well. If a user has chosen a destination but a sticker is scanned which is not along the route, a special message is presented and the route is recalculated to the nearest point of interest which matched the original destination. Note that if the point of interest, is, for example a lecture hall, and a user is now closer to a different entrance, this new entrance will be shown as the destination.

Extra consideration is taken when a user attempts to find a bathroom with the SOCS Wayfinder application. If a bathroom is full or out of order, a user can select to find a different bathroom. Since the user may have strayed off the intended path, they have to scan the closest sticker, and a new route will be calculated to next nearest bathroom.

\section{IMPLEMENTATION OVERVIEW}

Figure 5 gives an overview of the technologies used in the back-end implementation. In every instance, an open source and freely available library or technology was used. The back end is written in Java and the application for Android is written in Javascript, HTML, and CSS. Below, each technology is described, next, we describe how the back-end stack works as well as the Android application.

\subsection{SOCS Wayfinder Application}

Cordova: An open source framework from the Apache foundation [4] which allowed us to develop the application to eventually be used cross-platform (Android and iOS). Although many crossplatform frameworks are available, Cordova is arguably one of the oldest and most mature. Leaflet: An open source Javascript library 


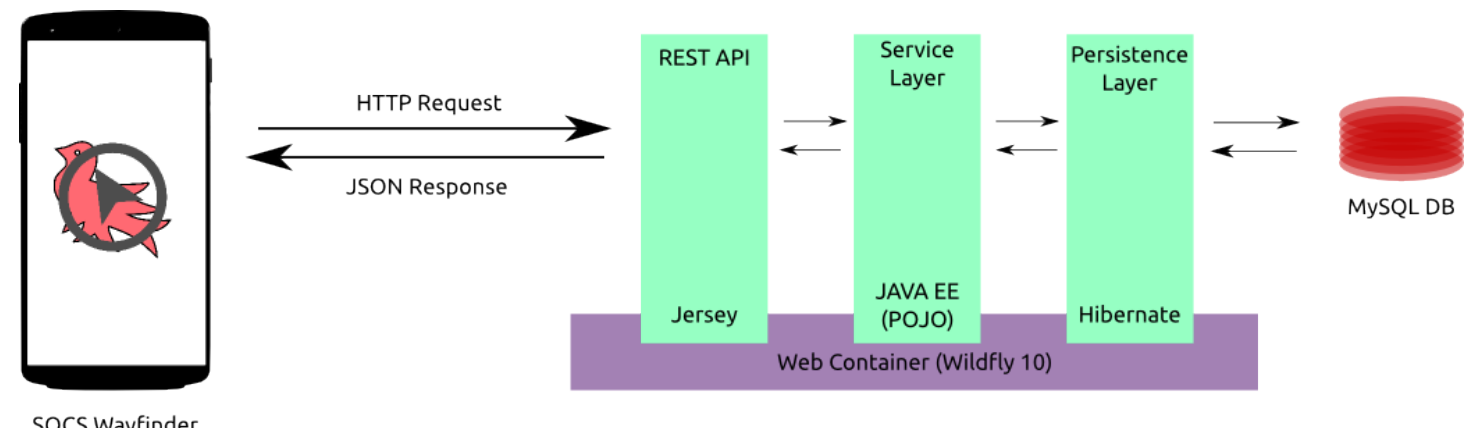

Fig. 5. Application and Back End Stack

for maps [3]. We use this library extensively in the application to show the current use location, routes, points of interest, and more. An open source library, as opposed to using an API like Google Maps was chosen since implementation in the hospital requires compliance with very specific privacy policy protocols. With open sourced libraries we can cut out the pieces that seem suspicious. Leaflet was chosen over some of the more extensive libraries such as OpenLayers since it was the smallest sized library that contained all of our required needs.

The Cordova application is built to heavily rely on making REST calls and hold very little data on the application itself. The images for the blueprints are preloaded in the application as it would be inconvenient to download when the user first starts the application. Upon loading the application, the NFC capabilities are enabled but blocked until the user finishes the tutorial, consent form and is on the scanning page. Scanning an NFC sticker retrieves the unique serial number from the sticker. Using a REST call, the serial number is matched to the Stickers table in our database. Since the associated floor is stored in the database, the image for the blueprints is loaded and the $(x, y)$ coordinates of the sticker position it over the blueprints. Should the server be unavailable, an error will pop up prompting the user to contact us for support.

From the next menu users can select the destination and another REST call verifies the closest point of interest using string comparison in the Stickers table. This retrieves all stickers which have the associated point of interest. The Dijkstra shortest path algorithm [8] is then run on the server to determine which sticker from the list is the closest. The route is returned to the mobile device and cached. The next REST call, and a call at each sticker during navigation returns the Navigation object which gives the direction to travel, as well as the humanized instructions between each steps.

\subsection{Back End}

Wildfly: An open source application server from Red Hat [7] which allows for development of web application. In our case, it serves more of a container to hold our REST API and make a connection to our database. Jersey: An open source RESTful framework provided by the Oracle Corporation [5], which helps develop REST applications. This is one of the best documented frameworks for Java. Hibernate: An open source persistence framework from Red Hat [6]. This allowed us to perform object relational mapping between our database and Java to allow us to treat our data as objects. This lets us leverage the combined power of Java and SQL rather than just one or the other.

The back end of the application is built around the data. Having the SQL database is an important feature of the back-end but it is not enough for the system to be effective. Using Hibernate to treat the data from different tables as objects allowed us to combine different fields and to create new objects from a combination of tables. A prime example was creating the Graph for the Dijkstra shortest path algorithm. Although the Navigation object, from the Navigation table in the database, gave us the starting and ending sticker, as well as the instructions, we didn't have the distance between the two stickers. By creating an edge object, we were able to include the Navigation object, as well as access the two sticker objects and evaluate the distance based on each $(x, y)$ coordinate.

The REST later interacts with the application in the scenarios above. Whenever a rest call is made, we verify that a secret key is sent to avoid security problems. After the key is verified, each field is type-checked and cast as necessary. No data is exchanged on the REST level. The service layer has can call and modify the data from the database. These calls are made by the persistence layer as a final verification for which objects can be called and serves as the initial recipient of data and can alert the server of errors. As such, we have a rigid system where only the specific queries we define can be run. The data which comes back from the service layer to the REST layer comes back in the JSON response which is sent to the mobile device. As such we have complete control over the data exchanged.

\section{GEOLOCATION}

The McConnell building is confusing for many new students and visitors. Firstly, not all floors have bathrooms for both men and women, so often people have to go searching for the proper bathroom on a different floor. Secondly, the two large lecture theatres span multiple floors and thus can be either the bottom or top, on different floors. Thirdly, the rooms are not numbered consecutively. Finally there are room numbers both in the form of 101 (main part of the floor) and $101 \mathrm{~N}$ (north annex of floor). These challenges motivated our SOCS Wayfinder application to create a simple and efficient way of letting a user find a specific point of interest.

\subsection{Choosing Sticker Locations}

Some areas on the building already had navigation signs put up by Professor Hendren and the Sable lab. There is an example of such a poster in Figure 6 Other areas of the building had no signs 
directing individuals to lecture theatres or bathrooms.
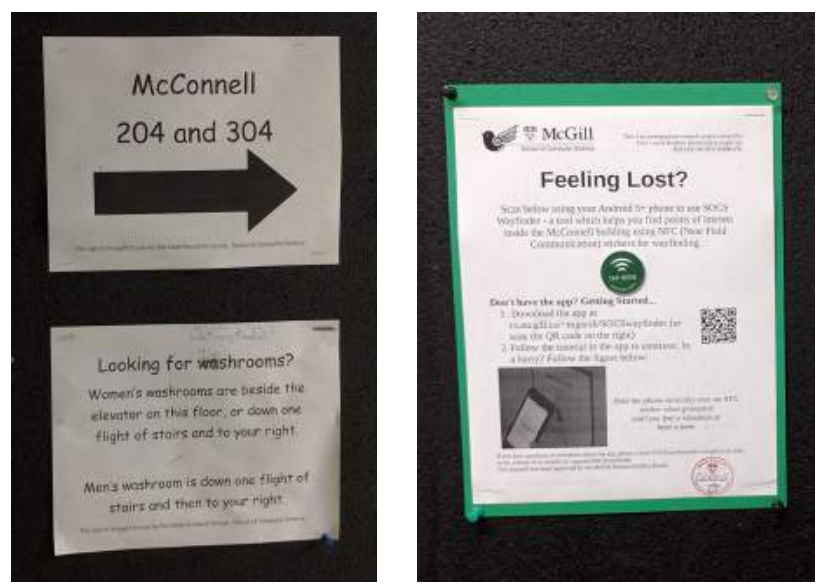

Fig. 6. An old navigation poster is shown on the left, with a new poster on the right.

The first step was to identify the location of all of the points of interest on our blueprints of the building. We noted all adjacent entrances to other buildings, bathrooms, administration offices, and lecture halls. Next, we differentiated between those accessible to users with special needs. Finally, we connected the points of interest along hallways, stairs, and elevators. In the case of hallways, any junction included a sticker. Any entrance to a stairwell or elevator also got a sticker. Finally, we have 25 points on the map, 15 of which are Stickers which are scannable, and the rest are points of interest. For locations where there were already paper navigation signs, we took a combined approach of having both the written instructions and the stickers. In other scannable locations, we have just the posters.

\subsection{Triangulation Position}

In preparation for using the blueprints in the application, each floor was resized and scaled to have the same width and height. Next, room numbers and other text was stripped to leave only the outline of the rooms, hallways, and stairwells. The chosen points of interest were assigned colours and filled in, receiving special annotations. Each colour was chosen specifically to be high contrast in order to allow users with various degrees of colour blindness to be able to use the application effectively. A second version of each floor was created with patterns rather than colours to further facilitate users.

In the database, each sticker has an $(x, y)$ coordinate location. This was chosen from the blueprints by calculating the distance from the bottom left corner. Each time a sticker is scanned by the application, the database returns the coordinates and the Leaflet library scales the coordinate to the proper location on the displayed map.

\section{PATHFINDING}

Once all of the stickers and points of interest were encoded into the database, we started to work on wayfinding between these stickers. Initially, we considered a simple breadth first search algorithm where we would build a graph of all of the locations, and simply search for the closest point of interest through the graph. However, we found that although the floors were similar in structure and size, some floors required fewer stickers. As such, our BFS algorithm would take us up and down flights of stairs more often than necessary. Instead, we implemented Dijkstra's shortest path algorithm using modified version of our Navigation object, to include distance between stickers, as an edge and Sticker objects as nodes. This gave us the shortest path from our source sticker to our destination sticker. We gave a penalty for whenever a navigation required changing floors, greater than the width and height of the blueprints, to discourage unnecessary floor traversals.

Figure 7 shows two nodes with an edge and the necessary information which is usually abstracted from the user. Each node (Sticker) has a unique identifier which allows us to change out an NFC sticker without modifying its symbolic meaning in our graph. It has a description for the researcher or database administrator, the floor that the sticker resides on, whether it is active, a timestamp, the unique serial number of the sticker, the associated $(x, y)$ coordinates, and whether it is accessible for users with special needs. Each edge (Navigation object and weight) consists of the two stickers - starting point and ending point. It has a flag whether the floor changes during the navigation between the two stickers, the instructions in english, whether it is accessible to users with special needs and the direction of the arrow on the screen. Each distance between two nodes is calculated by the distance between points using each node's $(x, y)$ coordinates on the blueprints.

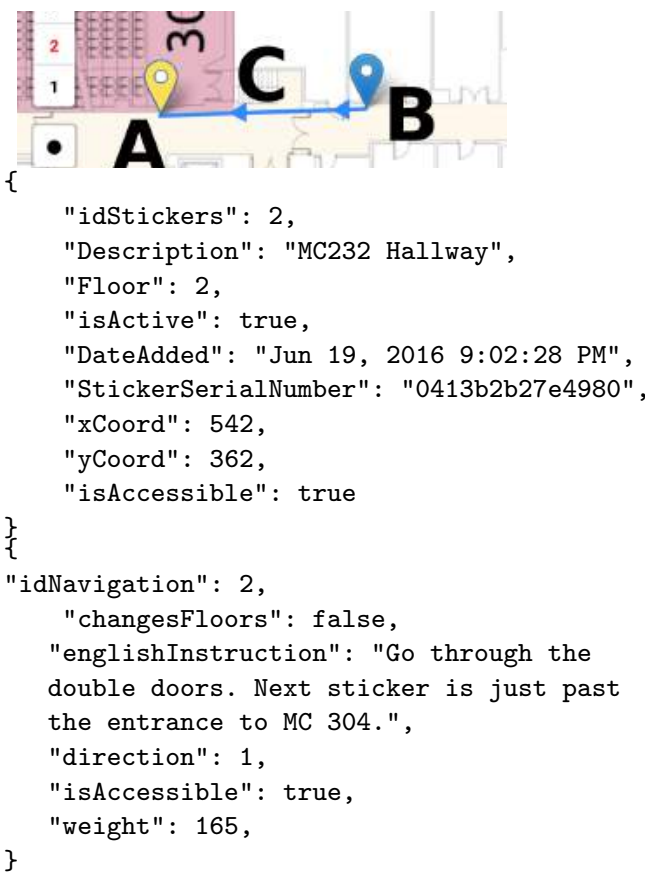

Fig. 7. An example of a node (A) and an edge (C) in our graph. Data which is usually abstracted from the user is presented in the table.

In order to account for users with special accessibility needs, the graph to search for the shortest path is built every time before running the algorithm. This is because a user can change their 
accessibility level at any time during using the application. When the graph is built, if a user has indicated the need accessible routes, any stickers which are not accessible points of interest, for example a classroom entrance with stairs or bathrooms without an accessible door are removed. For the edges, all stairwells are removed, as such, leaving only elevators to change floors.

In Figure 8 we present a step-by-step walkthrough of a simple navigational route. In Figure 9 we present a step-by-step walkthrough of a more complex navigational route with accessible options turned on.

\section{DATA COLLECTION}

When starting the application for the first time, after the user completes the tutorial and the consent form, there is a 2 question survey which is complete. We get the type of user (ie. Visitor, Student, Staff) and the general age group of the user. Alongside these 2 questions, the following gets stored about the user's device: manufacturer and model, operating system and the version, and unique identifier. This allows us to have information on who is using the application and whether we should provide more support for a specific size of device, or an operating system. The information collected cannot be traced to the actions the user takes in the application, such as scanning.

Whenever any user scans a sticker, we record the current sticker as well as the destination. When a user reaches the destination, or feels as though as they would like to stop navigating, they can give instantaneous feedback on their route. This allows us to build up a profile of the areas which are the most popular and effectively which may be the most confusing to newcomers. Furthermore, this allows us to analyze whether the directions we are giving to users are adequate.

\section{RELATED WORK}

Indoor navigation is a topic which has increased in popularity over recent years. As a result of more wide spread technologies such as WiFi and Radio-Frequency IDentification (RFID) tags and receivers of various calibers, much of the research has been focused on the triangulation of the users' position.

In 2007, Y. Chang et al. [9] presented a system which used geo-coded QR codes to assist individuals with cognitive impairments navigate indoors. When a user reached specific QR codes places throughout a building, a photo of the "just-in-time" direction is shown on screen. This helps the user navigate through visual and textual clues. Building upon this work, H. Hile et al. [11] evaluated a system where a user's camera on a phone would be used to determine landmarks and match them to predefined spaces. The purpose was also to aide individuals with cognitive impairments to navigate in indoor spaces. They were able to demonstrate that overlaying information on camera phones is feasible and with high accuracy but using image processing. In 2011, K. Curran et al. [10] evaluated indoor location determination technologies. They found that working with a RFID-radar system gave the researchers accurate locations, up to $50 \mathrm{~cm}$ in a specific area, and could track up to 50 users. However, this required the tags to be added to any object that needed to be tracked and required many receivers to cover a large area. Aside from the installation cost, this technology was more suited to applications where tags were stationary and would not work if the position needed frequent updating.

In 2015, D. Carboni et al. [12] studied different technologies for infrastructure-free indoor navigation. They found that WiFi based approaches are not satisfactory on their own. Even with a system that relied mostly on pre-existing WiFi networks for navigation, a dedicated set of access points would need to be installed for the system to work correctly. Aside from the financial cost of the equipment, it would need a technical to configure and reset the system periodically. However, tagging the environment with QR codes or RFID tags which are placed in reference points was found to be helpful in sensor-based indoor navigation.

An investigation using an NFC enhanced mobile device to find an optimal route to a selected destination was done in 2011 [13]. A user would arrive at a building with the application preloaded on their special enhanced device. A user would then scan NFC tags in order to navigate to a specific location.

In contrast to the above studies, the SOCS Wayfinder framework shows users humanized instructions between locations, measures data about usage, the maps can easily be manipulated to show multiple floors, the blueprints are preloaded on the application, it has accessibility options for users with limited mobility and colour blindness, it is readily available on the Android app store rather than only on specific NFC enabled devices, and uses a different implementation of Dijkstra's shortest path algorithm.

\section{CONCLUSIONS}

The main contributions of this project are: investigation of NFC stickers as viable geolocation tools, development of a robust platform for geolocation and wayfinder which is easily extensible, identifying confusing areas in the McConnell building which require an extra tool, designing map representations to allow for users with varying levels of colour blindness.

As a smaller-scaled implementation, this project was successful in identifying the potential obstacles when dealing with indoor geolocation. Some of these challenges included establishing a standardized method for identifying areas needing stickers or extra instructions. Another such challenge was to create an understandable and comprehensive interface which wouldn't require the user to invest too much time into learning the process and which they could start using right away.

With the ability to collect data on the types of users using the platform as well as the success of any navigation, we are able to ensure that the platform can grow and adapt as needed. Evolution becomes one of the biggest assets, especially in a project based on emerging technology. By building such a robust and extensible platform, this project represents only a small portion of the possibilities. As we discuss in the future work section, the addition of QR codes to include the Apple market, as well as older generation of phones is the next logical step.

\section{FUTURE WORK}

An immediate important evolution to this project would be the implementation of QR code scanning in order to capture devices which don't support NFC. Mainly, this would be required to involve the whole Apple market. Since the NFC scanning is just a small part of the SOCS Wayfinder application, and much of the data 

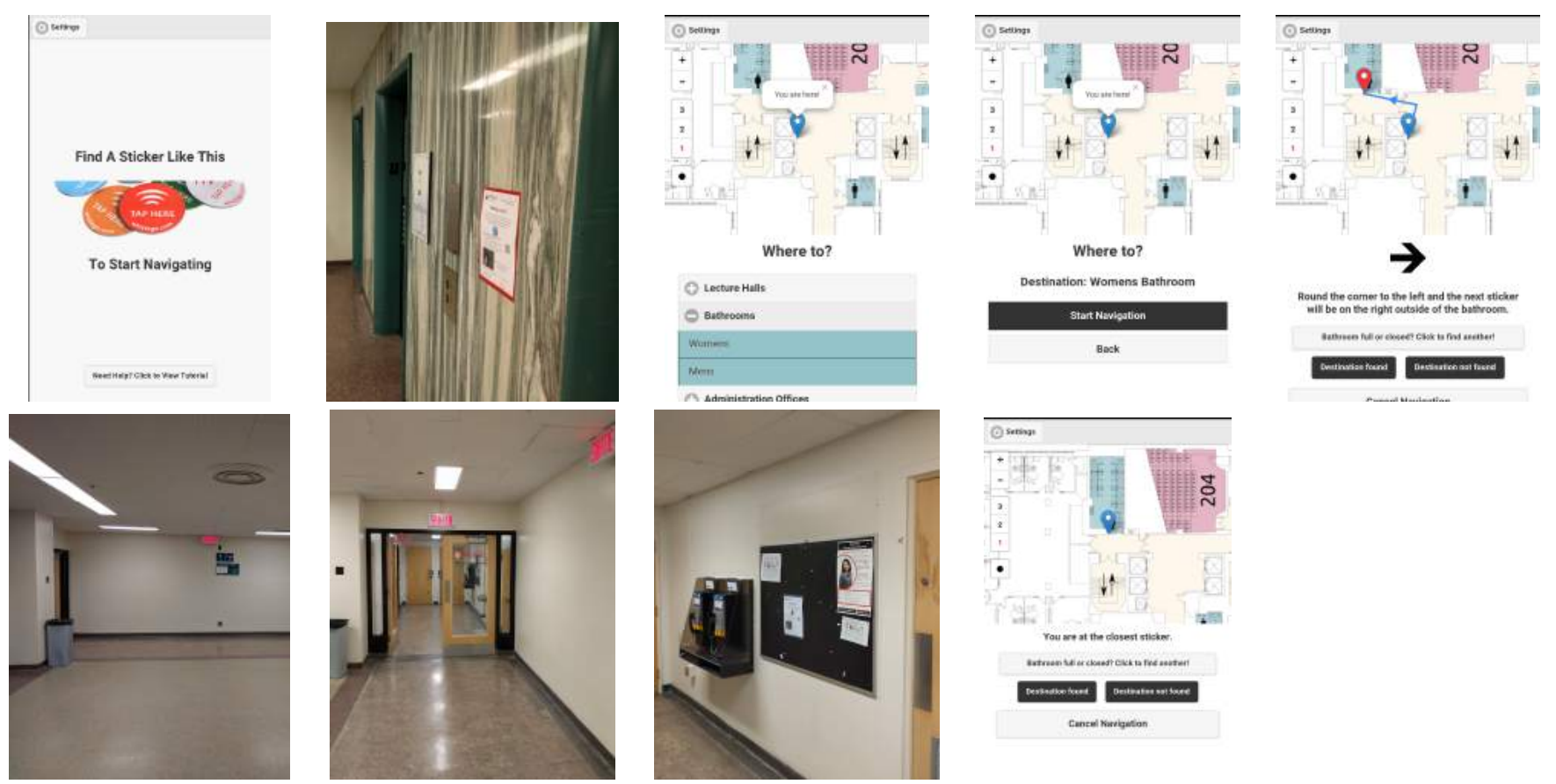

Fig. 8. An example of a simple navigation. A user opens the application to the scanning page and scans the closest sticker. The user selects their destination. Now, humanized instructions show the user where to go. Upon arrival at the destination, or anywhere along the route, the user can give feedback on their experience.

is transferred to and from the application using a REST service, the addition of QR codes will be relatively simple. The posters could also be modified in a way such that the QR code could be printed or pasted on top of an NFC sticker, keeping the instructions and the rest of the poster intact while adding support for many new devices.

Since this project was originally created with the Cedars Cancer Centre in mind, a next logical step would be to implement the technology described in this paper on a broader scale. By having access to blueprints of each floor, researchers could identify the main points of interest, break it down into categories and encode the coordinates in the database. Since the platform was built to be robust and universal, the data layer would not need to be changed for the relocation to the hospital. The new blueprints could be added directly into the application.

Having a geolocation and wayfinding tool is an asset to any public institution, even if it only caters to visitors or people new to a building. By extending the network of posters to include multiple buildings, the value of the tool would only grow. In the current form, the boundary between buildings is already well defined and marked as a point of interest. Since the Leaflet library supports tiling, other buildings which are added to the network could be loaded at run-time when a user is close to a border. By having a larger network, it would allow users to navigate between buildings instead of just between floors to efficiently reach a destination.

Since the application collects data on the type of users as well as the stickers scanned and the success of the navigation, it opens up opportunities to learn about the flow of users through specific areas of buildings. We can create heat maps of the most used locations and points of interest and see which areas of buildings may be the most confusing, requiring more posters to effectively guide users to their destination.

Finally, as technologies change rapidly, and since more people have access to more sophisticated and power smart-phone, a more lofty future direction would be to leverage more of these technologies. As we've discussed in related work, WiFi triangulation and fingerprinting may not be powerful enough to be an accurate or efficient choice of technology on its own. However, combining one of these techniques with NFC stickers, and the compass could give both a more accurate position, and provide more intuitive navigation. The NFC sticker could help to determine the initial location of the user, afterwards the WiFi and compass technologies could show a real-time position of the user on the blueprints as well as give a real-time arrow for how the user should proceed to the next sticker/next destination.

\section{REFERENCES}

[1] WhizTags. "NFC Done Beautifully." WhizTags. N.p., n.d. Web. 03 Aug. 2016.

[2] Tagstand. "How NFC Works." Tagstand. N.p., n.d. Web. 18 Jun. 2016.

[3] Agafonkin, Vladimir. "Leaflet - an Open-source JavaScript Library for Interactive Maps." Leaflet Dev Blog Atom. N.p., n.d. Web. 01 Aug. 2016.

[4] The Apache Software Foundation. "Get Started Fast." Apache Cordova. N.p., n.d. Web. 01 Aug. 2016.

[5] Oracle Corporation. "Jersey.” Jersey. N.p., 9 June 2016. Web. 01 Aug. 2016. 

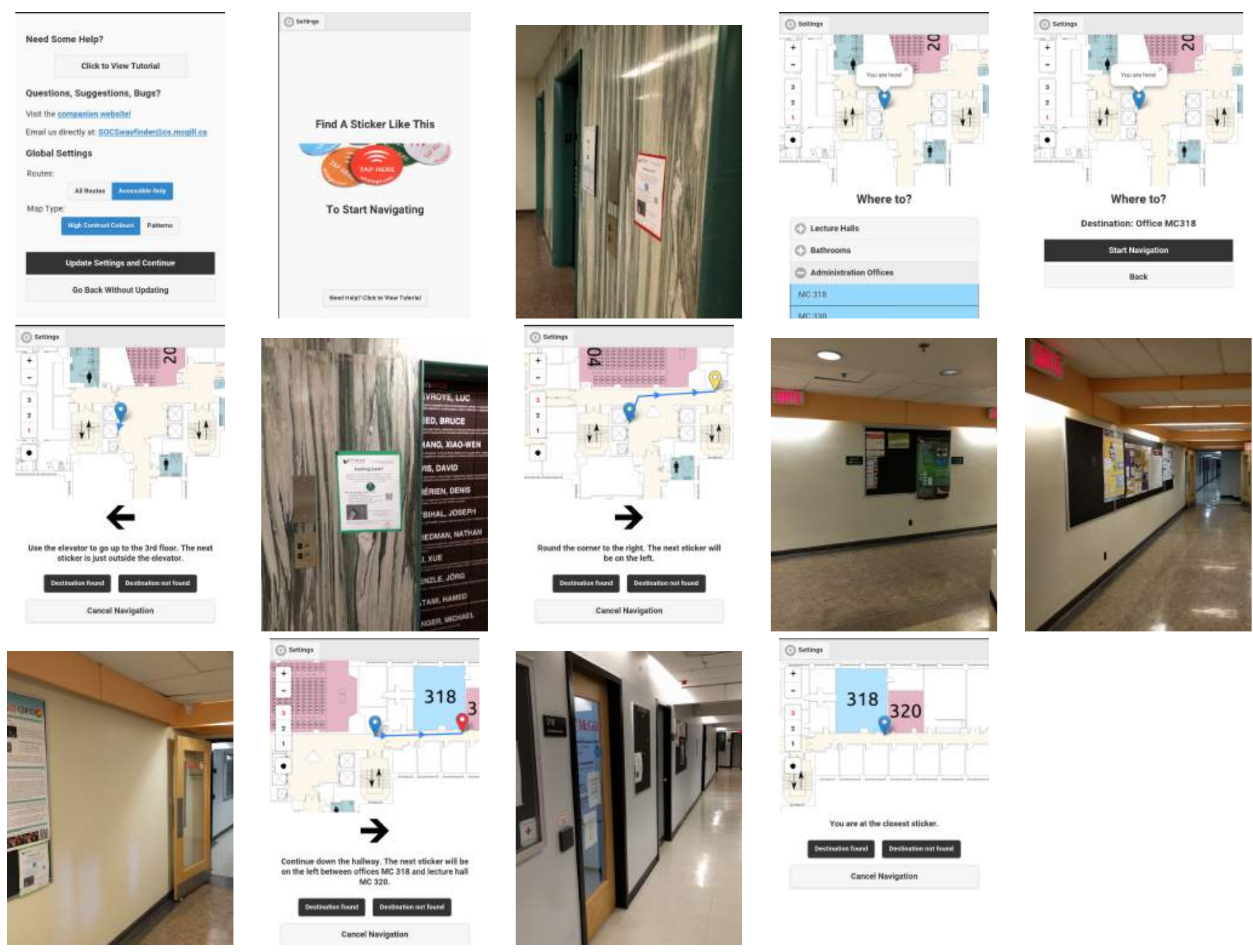

Fig. 9. An example of more complex navigation with an accessibility route. When a user has accessible routes turned on, the navigation to change floors suggests using the elevator. Much in the same way as changing floors with stairs, there is a sticker immediately outside of the elevator for simplicity. Once a user has navigated the section which requires accessible routes, in this case stairs, the rest of the route is the same.

[6] Red Hat, Inc. "Hibernate. Everything Data. - Hibernate." Hibernate. N.p., n.d. Web. 01 Aug. 2016.

[7] Red Hat, Inc. "WildFly.” Wildfly. N.p., n.d. Web. 1 Aug. 2016.

[8] Kleinberg, Jon, and Eva Tardos. "Greedy Algorithms." Algorithm Design. Boston, Mass.: Pearson/Addison-Wesley, 2014. 137-42. Print.

[9] Chang, Yao-Jen, Shih-Kai Tsai, Yao-Sheng Chang, and TsenYung Wang. "A Novel Wayfinding System Based on Geocoded Qr Codes for Individuals with Cognitive Impairments." Proceedings of the 9th International ACM SIGACCESS Conference on Computers and Accessibility - Assets '07 (2007): n. pag. Web.

[10] Curran, Kevin, Eoghan Furey, Tom Lunney, Jose Santos, Derek Woods, and Aiden Mccaughey. "An Evaluation of Indoor Location Determination Technologies." Journal of Location Based Services 5.2 (2011): 61-78. Web
[11] Hile, Harlan, and Gaetano Borriello. "Positioning and Orientation in Indoor Environments Using Camera Phones." IEEE Comput. Grap. Appl. IEEE Computer Graphics and Applications 28.4 (2008): 32-39. Web.

[12] Carboni, Davide, Andrea Manchinu, Valentina Marotto, Andrea Piras, and Alberto Serra. "Journal of Location Based Services." Infrastructure-free Indoor Navigation: A Case Study : Vol 9, No 1. Taylor \& Francis, 4 Mar. 2015. Web.

[13] B. Ozdenizci, K. Ok, V. Coskun, and M. N. Aydin, "Development of an indoor navigation system using NFC technology", in Proceedings of the fourth international conference on information and computing (ICIC), 2011 pp. 11-14. 